\title{
Can unstable relics save pure Cold Dark Matter?
}

\author{
A. Masiero, D. Montanino, and M. Peloso \\ Scuola Internazionale Superiore di Studi Avanzati (S.I.S.S.A.), \\ Via Beirut 4, I-34014 Trieste, Italy \\ and \\ Istituto Nazionale di Fisica Nucleare, Sez. di Trieste \\ Via Valerio 2, I-34127 Trieste, Italy
}

\begin{abstract}
The standard CDM model fails to describe the power spectrum of fluctuations since it gives too much power at small scales. Among other possible improvements, it has been suggested that an agreement with observations can be achieved with the addition of a late decaying particle, through the injection of non-thermal radiation and the consequent increase of the horizon length at the equivalence time. We analyze the possibility of implementing this idea in some extensions of the electroweak standard model, discussing the cosmological and astrophysical bounds to which these schemes are subject.
\end{abstract}

PACS number(s): 12.60.Jv, 14.60.St, 95.35.+d, 98.65.-r, 98.80.Cq

\section{INTRODUCTION}

The topic of structure formation in the early universe is still an open problem which has challenged many cosmologists in the past years. In the last decade great attention was devoted to the standard Cold Dark Matter (CDM) model, characterized by a flat universe with cold particles as dark matter, and by density fluctuations evolving from a primordial scale invariant (Harrison-Zel'dovich) spectrum.

This model turned out quite successful and its predictions about the properties of the different kinds of galaxies that form agreed well with observations [1]. However, more recent results [2.3] showed that, once normalized at large scale by COBE data, the standard CDM model predicts more power than observed at small scale. Several ideas have been proposed to improve the situation. A possibility is to change the inflationary prediction on the primordial spectrum. A non flat spectrum can be achieved for example in supergravity inflationary models [4], in models with more than one inflaton [5], or also by considering a non adiabatic evolution of the inflaton field [6. Another possibility is offered by mixed hot plus cold dark matter [7] and by "warm" dark matter models [8], since light particles have high speed at the decoupling era and cannot cluster on small scales.

Another way to fit the observations is the introduction of a non-zero cosmological constant $\Lambda$ [9]. At present this is the most appealing solution, since an incoming agreement about a cosmological constant is being provided by supernovae measurements [10,11]. By 
examining high-redshift SNe Ia and using them as candles to determine the distances to faraway galaxies, it is indeed possible to find evidence for an accelerating universe and thus exclude models with $\Omega_{\Lambda}=0$.

While statistics of these experiments is becoming more and more solid with the increasing of the number of observed high- $z$ supernovae (42 for [10] and 16 for [11]), still — as the authors of [10,11] say themselves — some doubts can be cast on their systematics. 1

Another point in favor of the option of a non-vanishing vacuum energy contribution comes from measurements of $\Omega_{\text {matter }}$ and $\Omega_{\text {total }}=\Omega_{\text {matter }}+\Omega_{\Lambda}$. The latter quantity can be observationally determined from the position of the first acoustic peak of the Cosmic Background Radiation (CBR). Preliminary results (which are still plagued by large errors) indicate a preference for $\Omega_{\text {total }} \simeq 1$ (see [13] and references therein). Clearly, also theoretical arguments based on inflation strongly favor a flat universe. As for $\Omega_{\text {matter }}$, its best determination comes from measurements of the baryon fraction in clusters. Using clusters as "fair samples" of matter in the universe, the bound on $\Omega_{\text {baryons }}$ from the standard nucleosynthesis, and determinations of the amount of baryons and the total matter in clusters, it has been inferred that $\Omega_{\text {matter }}$ should not exceed $40 \%$ [14]. I As convincing as this arguments look for $\Omega_{\text {matter }}<1$, we think that they should not be considered conclusive on the intriguing issue of the matter present in the universe.

In view of the present uncertainties, we think it is still valuable to consider other options tackling the failure of the standard CDM model. In this paper we focus on the possibility that, in addition to the CDM, there exist decaying (or sometimes called volatile) particles. The decay products of these particles can be used to increase the horizon length at the equivalence time, hence increasing the power at large scales [15, 16].

In the past years, only a couple of schemes which implement this idea have been proposed (see for example [15 [18]). Indeed, the candidate models must have among the decay products only very weakly interacting particles, since stringent cosmological and astrophysical bounds apply when more interacting particles are produced. The most popular scenario for the realization of the shift of $\lambda_{\text {eq }}$ involves an $m \sim \mathrm{MeV}$ neutrino decaying in majoron plus a lighter neutrino. An alternative possibility is given by decays with the presence of the axion field and its superpartners.

The aim of the present paper is to update and extend the above analyses in light of some other candidate models and of the most recent observational data. The main new possibility

\footnotetext{
${ }^{1}$ It is worthwhile to remark here that the introduction of a cosmological constant opens many other unsolved puzzles. First of all, if one associates the cosmological constant to the energy of the vacuum, one would expect either $\Omega_{\Lambda}=0$ (if some symmetry prevents it) or $\Omega_{\Lambda} \sim M_{\mathrm{pl}}^{4} / \rho_{\text {critical }}$, while $\Omega_{\Lambda}=\mathrm{O}(1)$ appears as an incredibly fine-tuned small value. Second, it is not clear why $\Omega_{\Lambda}$

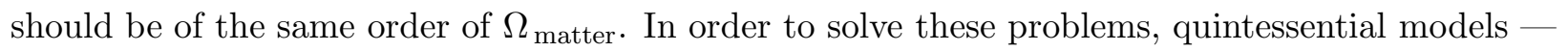
where $\Omega_{\Lambda}$ is a dynamical parameter associated to the energy of a slow evolving scalar field — are becoming popular, but they are far from being conclusive [12].

${ }^{2}$ Other circumstantial evidences for $\Omega_{\text {matter }}<1$ related to the evolution of the abundance of rich cluster with redshift are more controversial.
} 
that we suggest involves decays with production of high energy neutrinos. These processes must be handled with care since they can enter in conflict with bounds imposed by the non observations of the neutrinos (mainly for very late decays) or (for early enough decays) by Big-Bang Nucleosynthesis. These last bounds apply because the produced neutrinos can scatter off background antineutrinos, generating an electromagnetic cascade which can photodissociate the light elements previously produced.

The work is organized as follows. In Sec. II we present the general idea of the shift of the equivalence scale. In Sec. III we report the main cosmological and astrophysical bounds which apply in case of production of high energy neutrinos. In Sec. IV we discuss the possibility to implement this idea in a supersymmetry context. In Sec V we switch to the option involving $m \sim \mathrm{MeV}$ unstable neutrinos and we study two other decaying processes (namely the decay into three neutrinos and into neutrino plus familon) in addition to the more common decay into neutrino plus majoron. The results and some prospects for the future are presented in the conclusions.

\section{SHIFT OF THE EQUIVALENCE SCALE}

As we said in the introduction, the standard CDM model fails to describe the observed spectrum of fluctuations. However, an agreement with observations can still be reached in an $\Omega_{\mathrm{CDM}+\text { baryons }}=1$ context if the horizon length at the equivalence scale is increased. The reason is that during the radiation dominated era super horizon fluctuations can grow, while sub horizon ones can not. Thus, if we delay the matter domination (or, in other words, we increase the horizon length $\lambda_{\text {eq }}$ ) we give the large modes more time for growing, while the small (sub horizon) ones are kept frozen by a sort of "pressure of radiation".

In standard models (with relativistic particle at present provided by CBR photons and three species of relic neutrinos) the length of the horizon at the equivalence time is given by

$$
\lambda_{\mathrm{eq}} \simeq 30\left(\Omega h^{2}\right)^{-1} \mathrm{Mpc}
$$

where $\Omega$ is the ratio density of the universe over critical density ( $\Omega=1$ for a flat universe) and $h$ is the Hubble parameter in units of $100 \mathrm{Km} \mathrm{sec}^{-1} \mathrm{Mpc}^{-1}$. Inflationary models strongly prefer $\Omega=1$, while observations constrain $h \in[0.4,1]$. P On the other hand, the observed spectrum of fluctuations is well fitted by CDM models only for

$$
\lambda_{\mathrm{eq}} \simeq 150 h^{-1} \mathrm{Mpc}
$$

i.e., for $\Omega h=0.2$.

Instead of lowering $\Omega h$, a raising of $\lambda_{\text {eq }}$ can be achieved by considering an unstable matter which decays into relativistic particles [15, 16]. Photons cannot accomplish this task:

\footnotetext{
${ }^{3}$ More recent data suggest to tighten this interval for $h(h=0.67 \pm 0.10$ [19]). However, since a stable agreement on the value of the Hubble constant has not been reached so far, in our analysis we prefer to stick to the more conservative range $[0.4,1]$.
} 
indeed $\gamma$ produced after $t \simeq 10^{6}$ sec must be of negligible amount or they would distort the shape of the CBR (see next section), while photons emitted at early times thermalize and just contribute to rising the temperature of the (thermal) background to the value we measure today [and from which we get Eq. (互)]. If the decay products are not photons (let us call them "invisible"), the result is that the relativistic energy is greater than the one that we infer just measuring the temperature of the CBR. Thus, for appropriate choice of the decay time $\tau, \lambda_{\text {eq }}$ can increase.

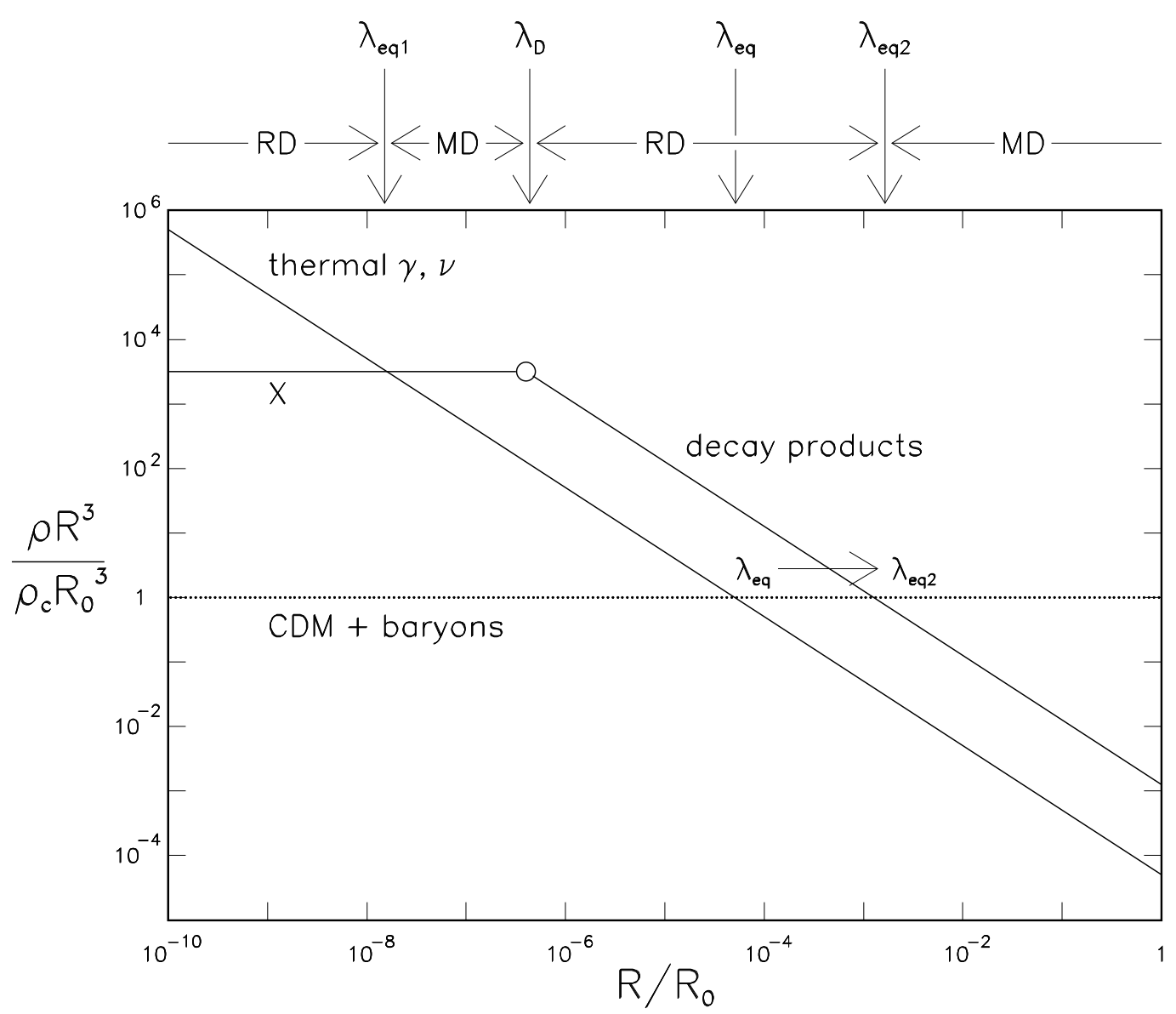

FIG. 1. Schematic representation of the model. RD and MD denotes, respectively, Radiation and Matter Domination. See the text for other details.

In Fig. 1 (inspired by the work of H.B. Kim and J.E. Kim [16]) we give a schematic representation of the model that we have described.

We denote by $X$ the matter which decays, by $m Y_{\text {tot }}$ its (comoving) energy density, f

\footnotetext{
${ }^{4}$ Following the standard notation, $m$ is the mass of the particle, while $Y_{\text {tot }}$ is the ratio between
} 
and by $m Y_{\text {rel }}$ the part of it which goes into relativistic particles. f

The $X$ particle is not relativistic before decaying, thus its energy density increases with respect to the thermal one. In order to realize the shift of $\lambda_{\text {eq }}$, we see from Fig. 1 that $X$ must decay only after it dominates. Thus the main peculiarities of this scheme are (i) that $\lambda_{\text {eq }} \rightarrow \lambda_{\text {eq2 }}>\lambda_{\text {eq }}$, and (ii) that a new matter domination era - starting at $\lambda_{\text {eq } 1}$ - arises. The values of these two lengths are [16]

$$
\begin{aligned}
& \lambda_{\mathrm{eq} 1} \simeq 8 \cdot 10^{-2} \mathrm{Kpc}\left(\frac{\mathrm{MeV}}{m Y_{\mathrm{tot}}}\right) \\
& \lambda_{\mathrm{eq} 2} \simeq 30 \mathrm{Mpc}\left(\Omega h^{2}\right)^{-1}\left[\left\{\frac{1}{0.55}\left(\frac{\tau}{\mathrm{sec}}\right)\left(\frac{m Y_{\mathrm{rel}}}{\mathrm{MeV}}\right)^{2}\right\}^{2 / 3}+1\right]^{1 / 2} .
\end{aligned}
$$

The scheme is implemented if $\lambda_{\text {eq2 }} \simeq 150 h^{-1} \mathrm{Mpc}$ [cfr. Eq. (2)], namely:

$$
\left(\frac{\tau}{\mathrm{sec}}\right)\left(\frac{m Y_{\mathrm{rel}}}{\mathrm{MeV}}\right)^{2} \simeq 0.55\left[(h / 0.2)^{2}-1\right]^{3 / 2}
$$

For the model to phenomenologically hold, some bounds have to be respected. Those stated here are somehow model independent and represent general constraints that have always to be taken into account. In the next section we will instead discuss limits which apply in case of production of high energy neutrinos.

The first bound comes from nucleosynthesis and requires that at the time of neutrino decoupling the energy density of $X$ is less than that of one neutrino species [20]:

$$
\left(\frac{m Y_{\mathrm{tot}}}{\mathrm{MeV}}\right)<0.107
$$

The demand that the fraction of the energy density into relativistic decay products is high enough to realize the shift yields:

$$
\left(\frac{m Y_{\text {rel }}}{\mathrm{MeV}}\right)>3.6 \cdot 10^{-6} h^{2}
$$

Notice that imposing Eqs. (3), (4), and (5), we safely avoid overclosure of the universe by the decay products.

its number density and the entropy density of the universe.

${ }^{5}$ For example, in Sec. III we will discuss the decay gravitino $\rightarrow$ sneutrino + neutrino. The sneutrinos produced by this process have a mass which is typically of the same order of the one of the gravitinos, and thus they quickly derelativizes. Hence, the only energy which contributes to the shift of $\lambda_{\text {eq }}$ and which we denote by the suffix rel is the one associated to the neutrinos. Thus, in this case $m Y_{\text {rel }} \sim m Y_{\text {tot }}$. 


\section{COSMOLOGICAL AND ASTROPHYSICAL BOUNDS}

As already remarked, the bounds considered in the previous section are certainly necessary, but they can be not sufficient if the decay products affect our present observations in some other ways than the only shift of the equivalence scale. [0

For example, severe limits exclude the possibility of realizing the shift of $\lambda_{\text {eq }}$ if the decay produces a non negligible amount of photons. I Indeed, Eq. (3) is satisfied only if the relativistic energy density is about 25 times [16] larger than the thermal one (that is the one that we infer by measuring the temperature of the microwave background radiation). As a consequence, if the decay occurs at early time, the produced photons thermalize and thus must not carry more than $1 / 25$ of the total energy density of the decaying particles. On the other hand, if the decay happens later the photons produced must be much less than the thermal ones, or they would appear as a clear distortion in the observed black-body spectrum. In both cases, it follows that the amount of energy density in form of photons must be much less than the one undergone into other relative particles.

From the above discussion we realize that the shift of $\lambda_{\text {eq }}$ cannot be implemented in decays with the production of photons. We thus switch to the case of high energy neutrinos among the decay products, which needs a more detailed discussion. In fact, in this case one has to discuss some limits which come from the non observation of the neutrinos and from BBN.

- Non observation: The limits coming from the IMB nucleon decay detector are low enough [21,22] \& to rule out a flux of neutrinos necessary to shift $\lambda_{\text {eq }}$ if they have at present an energy $E_{\nu_{0}}>100 \mathrm{MeV}$.

The energy interval $1.5 \mathrm{MeV}<E_{\nu_{0}}<100 \mathrm{MeV}$ has been in the past or it is currently monitored by several experiments 24 26] and no claim has been made about an anomalous diffuse neutrino flux.

We thus impose the safe requirement $E_{\nu_{0}}<1 \mathrm{MeV}$, i.e. that neutrinos coming from the decay of the $\mathrm{X}$ matter do have a present energy lower than $1 \mathrm{MeV}$.

Making the approximation $E_{\nu_{0}} \simeq\left(\tau / t_{\text {eq }}\right)^{1 / 2}\left(t_{\text {eq }} / t_{0}\right)^{2 / 3} E_{\nu}$, where $E_{\nu}$ is the energy of the neutrinos at their production, we get

$$
\frac{E_{\nu}}{\mathrm{GeV}}<4 \cdot 10^{6}\left(\frac{\mathrm{sec}}{\tau}\right)^{1 / 2}
$$

\footnotetext{
${ }^{6}$ The bounds (4) and (5) are sufficient for processes like axino $\rightarrow$ axion + gravitino [16], or for decays with production of low energy neutrinos, since all these particles have very weak interactions.

${ }^{7}$ The authors are grateful to S. Bonometto for remarking this point.

${ }^{8}$ See also [23], where the specific case of a decaying fermion with abundance determined by annihilation via Standard Model gauge bosons is considered.
} 
We see that, for a given value of energy density of the produced neutrinos $m Y_{\nu}$, decays with lower values of the energy of each neutrino (and higher numerical density) are less constrained.

- BBN : neutrinos produced at early times can annihilate off background antineutrinos and produce $e^{ \pm}$pairs; then the charged particles can photodissociate - via electromagnetic cascade — the light elements produced during BBN [27,28].

In the limit of Fermi approximation g the fraction of neutrinos "converted" into radiation is 28

$$
f_{\nu \rightarrow \gamma} \simeq 1.3 \cdot 10^{-3}\left(\frac{E_{\nu}}{\mathrm{MeV}}\right)\left(\frac{\mathrm{sec}}{\tau}\right)
$$

Thus, once the neutrinos from the decay have enough energy to generate $e^{ \pm}$pairs, $\square$ we must calculate the density of radiation produced

$$
(m Y)_{\gamma}=(m Y)_{\nu} \cdot f_{\nu \rightarrow \gamma},
$$

and analyze if it can spoil the good agreement between the observational data and the Standard Big-Bang Nucleosynthesis (that is in absence of decaying particles). In this way we can get an upper limit on $f_{\nu \rightarrow \gamma}$ as a function of the decay lifetime $\tau$.

We do not review here the whole analysis about the impact of an electromagnetic cascade on the abundance of the light elements, but we just state the results of the numerical work [29], addressing the interested reader to the references listed there. $\square$

The two primordial abundances that most constrain the theoretical predictions are the ${ }^{4} \mathrm{He}$ and the deuterium ones. At present, different observations give the amount of these abundances with some discrepancies:

$$
\begin{array}{rll}
\text { Low D } & : & y_{2}=(3.39 \pm 0.25) \cdot 10^{-5}, y_{2}=n_{D} / n_{H} \\
\text { High D } & : & y_{2}=(1.9 \pm 0.5) \cdot 10^{-4} \\
\text { Low }{ }^{4} \mathrm{He} & : \quad Y=0.234 \pm(0.002)_{\text {stat }} \pm(0.005)_{\text {syst }} \quad, \quad Y=\frac{\rho^{4} \mathrm{He}}{\rho_{\text {baryons }}} \\
\text { High }{ }^{4} \mathrm{He} & : \quad Y=0.244 \pm(0.002)_{\text {stat }} \pm(0.005)_{\text {syst }}
\end{array}
$$

\footnotetext{
${ }^{9}$ Valid for $E_{\nu} E_{\bar{\nu}}<M_{Z}^{2}$, i.e.,
}

$$
\left(\frac{E_{\nu}}{\mathrm{GeV}}\right)\left(\frac{\mathrm{sec}}{\tau}\right)^{1 / 2}<10^{7}
$$

that is for all cases of our interest.

$$
{ }^{10} \text { i.e., for }\left(\frac{E_{\nu}}{\mathrm{MeV}}\right)\left(\frac{\mathrm{sec}}{\tau}\right)^{1 / 2}>0.3
$$

\footnotetext{
${ }^{11}$ See also [30], where a detailed analysis including electromagnetic cascade has been performed for lifetime bigger then $10^{15} \mathrm{sec}$.
} 
We discuss the implications considering the different above ranges.
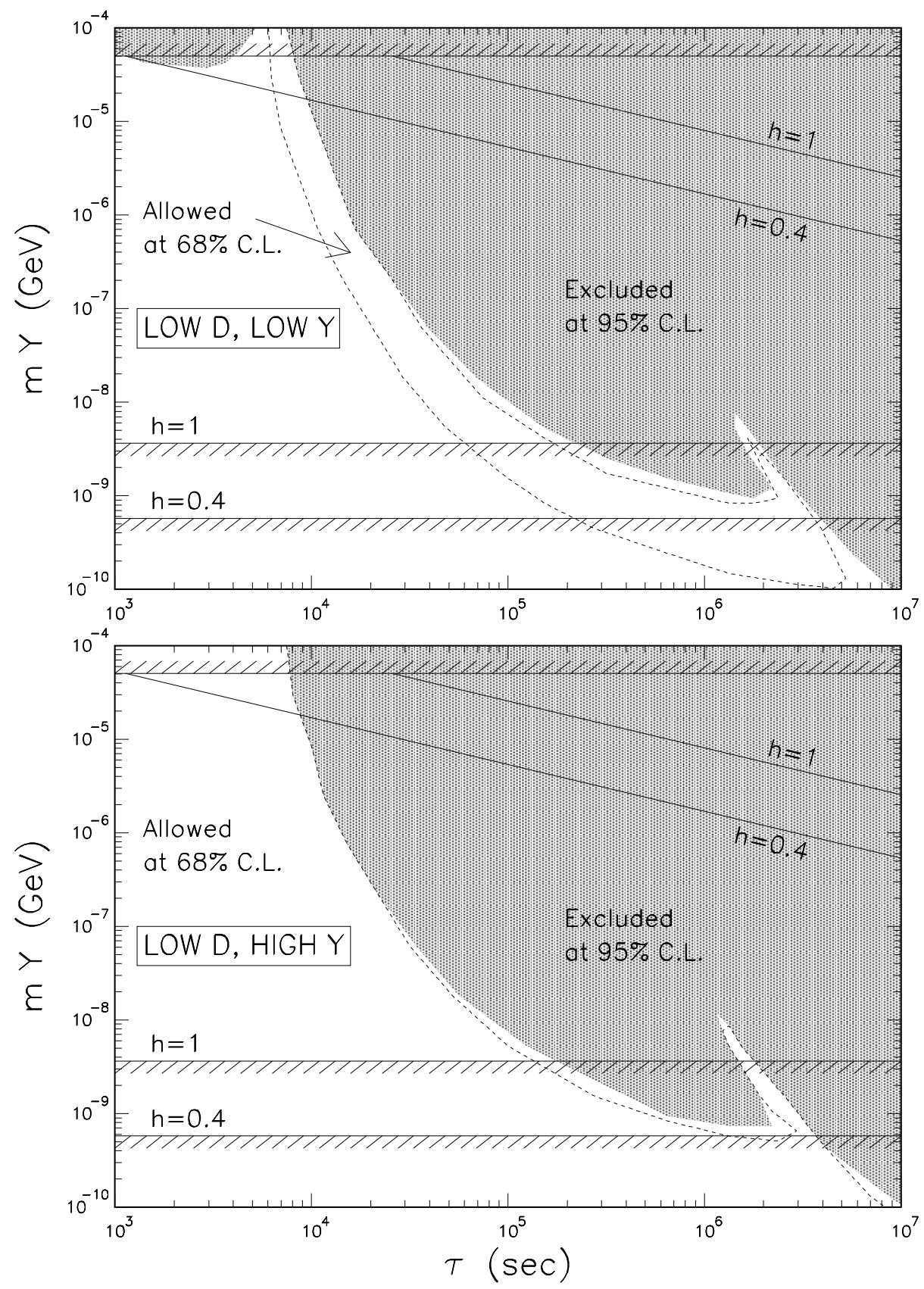

FIG. 2. Bounds on an electromagnetic cascade in the case of low D and ${ }^{4} \mathrm{He}$ (upper panel) low D and high ${ }^{4} \mathrm{He}$ (lower panel). Gray area: excluded zone at $95 \%$ C.L. by nucleosynthesis; dotted line: limit at $68 \%$ C.L. by nucleosynthesis. See the text for details.

In the case of low $\mathrm{D}$ and low ${ }^{4} \mathrm{He}$, the predictions of the standard BBN (i.e. without decaying particles) are excluded at $68 \%$ confidence level. A non-vanishing $m Y_{\gamma}$ is preferred in order to destroy a sufficient amount of the primordial deuterium. Thus, if 
this case will turn out to be the right one, it could be worth to examine if the scheme that we are proposing here can be advocated both to realize the shift of $\lambda_{\text {eq }}$ and to explain the would be "standard BBN failure".

The other three cases give qualitatively similar results. For all of them standard BBN is allowed and we have upper bounds on $m Y_{\gamma}$ for each value of $\tau$.

The results are summarized in Fig. 2. First we report (from [29]) the bounds imposed by BBN on $m Y_{\gamma}$. We show only the case of low $\mathrm{D}$ and low ${ }^{4} \mathrm{He}$ and the case of low $\mathrm{D}$ and high ${ }^{4} \mathrm{He}$ (the other two being similar to the last one). The gray area denotes the points in the plane $m Y_{\gamma}$ vs $\tau$ excluded at $95 \%$ C.L. The region within the dotted lines corresponds to points allowed at $68 \%$ C.L. (in the lower panel these regions extends over the entire blank space).

Then we analyze the impact on these regions of the implementation of the shift of $\lambda_{\text {eq }}$ with a decay in high energy neutrinos with the subsequent production photons. The straight lines refer to a decay in which the initial energy density is equally shared between neutrinos and a massive decay particle which quickly derelativises $\left(m Y_{\nu}=\right.$ $\left.m Y_{\text {rel }}=\frac{1}{2} m Y_{\text {tot }}\right)$. The horizontal lines correspond to the upper and lower bounds $m Y_{\text {tot }}<0.107 \mathrm{MeV}$ [Eq. (困)] and $m Y_{\nu}>3.6 \cdot 10^{-6} h^{2} \mathrm{MeV}$ [Eq. (5)], while the two diagonal ones indicate for each $\tau$ the amount of $m Y_{\nu}$ produced when the shift of $\lambda_{\text {eq }}$ is realized [Eq. (3)].

The results shown in Fig. 2 put a severe constraint on $f_{\nu \rightarrow \gamma}$, since they show that the amount of neutrinos "converted" in photons must be a very small fraction of the total one in order to respect the limits imposed by BBN. For example, we see that at $\tau=10^{7}$ sec the quantity $f_{\nu \rightarrow \gamma}$ must be smaller than about $10^{-5} \div 10^{-4}$ (according to different values of $h$ ). 田

In the next section we study if it is possible to implement the shift of $\lambda_{\text {eq }}$ in supersymmetric models with and without $R$ parity. The main part is devoted to the study of two processes with the production of high energy neutrinos. We show there that the only one of the two model which is workable satisfies also the bounds discussed in this section.

\section{SUPERSYMMETRIC DECAYS}

In supersymmetric models, the terms

$$
\lambda_{i j k} L_{i} L_{j} E_{k}^{c}+\lambda_{i j k}^{\prime} L_{i} Q_{j} D_{k}^{c}+\mu_{i} L_{i} H_{u}+\lambda_{i j k}^{\prime \prime} U_{i} D_{j} D_{k}^{c}
$$

are usually dropped away from the superpotential in order to avoid fast proton decay. To do this, a discrete symmetry which is called $R$ parity and which is violated by the above terms is imposed.

\footnotetext{
${ }^{12}$ The same limit must be imposed also on the branching ratio of the decay channels in which photons are produced.
} 
$R$ parity forbids terms in which supersymmetric particles are in odd number. As a consequence, superparticles can be produced or destroyed only in pairs, and the Lightest SuperParticle (LSP) is stable. An advantage of supersymmetry is that this particle, for adequate but natural choices of the Minimal Supersymmetric Standard Model (MSSM) parameters, is a good cold dark matter candidate [31].

However, proton decay requires the violation of both baryon and lepton number. Thus it is not in disagreement with the present limits on proton lifetime to make vanishing the last term in Eq. (9) while keeping different from zero some (or all) of the first three, or viceversa to take only the last term non-vanishing.

Another phenomenologically acceptable possibility is to break $R$ parity spontaneously. In these scenarios one typically introduces some isosinglets which acquire a $V E V$ and break $R$ and Lepton symmetries, without entering in conflict with the bounds imposed by the $Z^{0}$ width.

Whatever possibility one may choose, as a result in both cases the LSP is non longer stable and, unless we take very a very small breaking of $R$ [32], it can not be invoked as CDM. Nevertheless, it may be possible for its decay to implement the shift of $\lambda_{\text {eq }}$.

In the next two subsections we discuss the possibility of realizing the shift of $\lambda_{\text {eq }}$ in a supersymmetric context, first in schemes with conserved $R$ parity and then in others where this symmetry is broken.

\section{A. Conserved $R$ parity}

In the context of supersymmetry with conserved $R$ parity one would like to implement the shift of the equivalence scale through the decay of the next LSP to the LSP. The main difficulty is that "ordinary" superparticles decay extremely fastly. For example, the lifetime of the decay $\tilde{\nu} \rightarrow \tilde{B} \nu$ is

$$
\tau \sim 10^{-24} \sec \left(1-\frac{m_{\tilde{B}}^{2}}{m_{\tilde{\nu}}^{2}}\right)^{-2},
$$

and a strong fine tuning is required in order to have a late decay.

It is thus mandatory to consider processes with very low interacting particles. One of these particles is the gravitino (that is the spin $3 / 2$ superpartner of the graviton) since its interactions are suppressed by inverse powers of the Planck mass.

This option is already present in [16], where the decay axino $\rightarrow$ axion + gravitino is considered. This scheme implements the shift of $\lambda_{\text {eq }}$ for several values of the axino decoupling temperature with respect to the reheating temperature, and its good "flexibility" is essentially due to the very little danger that both the produced particles have on our observations.

Another interesting scheme involving an unstable gravitino is present in [33], where the scenario characterized by the process ${ }^{13}$ gravitino $\rightarrow$ neutrino + sneutrino is discussed in

\footnotetext{
${ }^{13}$ We do not consider here other decay modes of the gravitino into "ordinary" superparticles, since they typically produce photons.
} 
details, but without considering the problem of the shift of the equivalence scale.

The scheme proposed in [33] is a very interesting one, since it has the following advantages:

- In standard models either $m_{3 / 2} \leq \mathrm{keV}$ (if stable) or $m_{3 / 2} \geq 10 \mathrm{TeV}$ (if it decays, this bound is imposed by BBN). Here the primordial gravitinos are diluted away by inflation, and the ones considered are generated by the reheating. For appropriate values of the reheating temperature, intermediate masses are allowed [34].

- Usually the sneutrino is not assumed as the LSP, since its relic abundance is very small. In this case we have instead $Y_{\tilde{\nu}}=Y_{3 / 2}$.

- There is absence of direct $\gamma$ production.

The following relations hold [34]:

- abundance from the reheating:

$$
m Y_{3 / 2}=2.6 \cdot 10^{-8} \mathrm{GeV}\left(\frac{T_{r}}{10^{13} \mathrm{GeV}}\right)\left(\frac{m_{3 / 2}}{100 \mathrm{GeV}}\right) \quad ;
$$

- decay lifetime:

$$
\tau=3.92 \cdot 10^{8} \sec \left(\frac{100 \mathrm{GeV}}{m_{3 / 2}}\right)^{3}\left(1-\frac{m_{\tilde{\nu}}^{2}}{m_{3 / 2}^{2}}\right)^{-4}
$$

- Fraction of energy transferred to the neutrino:

$$
F=\frac{1}{2}\left(1-\frac{m_{\tilde{\nu}}^{2}}{m_{3 / 2}^{2}}\right) \quad
$$

- Closure by $\tilde{\nu}$ :

$$
\left(\frac{m_{\tilde{\nu}}}{100 \mathrm{GeV}}\right)\left(\frac{T_{r}}{10^{13} \mathrm{GeV}}\right)=0.14 h^{2} \Omega_{\tilde{\nu}}
$$

In these equations, $T_{r}$ is the reheating temperature and $\Omega_{\tilde{\nu}}$ is the contribution to the critical density of the sneutrinos from the decay. We ask for $\Omega_{\tilde{\nu}} \simeq 1$.

The conservative experimental bound $m_{3 / 2}>m_{\tilde{\nu}} \geq 43 \mathrm{GeV}$ [35] limits [from Eq. (14)] $T_{r}$ and thus the abundance $m Y_{3 / 2}$. It follows that, when we try to implement Eq. (3), we must delay $\tau$ by a fine tuning $m_{\tilde{\nu}} \simeq m_{3 / 2}$. However, in this case the fraction $F$ of energy to the neutrinos become very low and the bound $m Y_{3 / 2} F>3.6 \cdot 10^{-6} h^{2} \mathrm{MeV}$ is never satisfied (in other words, we have neutrino domination only if we delay the decay after the allowed time). 
We can ask how we can improve the situation. One possible way is to require that the sneutrino is not the LSP and thus the ones produced by gravitino decay go in turn into neutrino plus neutralino (now the LSP). In this case we can expect an improvement since, even with fine tuning, the second neutrino has about half the energy of the initial gravitino.

In order for this scheme to work, the direct decay gravitino $\rightarrow$ neutralino + its ordinary partner must be forbidden by kinematics, or it would be the dominant channel (with consequent production of photons). The best that we can do is to choose $\chi=$ pure higgsino and forbid the direct decay by suitably rising the mass of the corresponding higgs particle. T4 However, it turns out that this scheme requires $m_{3 / 2}=\mathrm{O}(\mathrm{TeV})$ and thus raising the higgs mass at this scale appears rather unnatural.

A possible solution is to consider the inverse process with the sneutrino as NLSP which decays into the gravitino plus neutrino. The first problem is the possible overclosure of the universe by the relic gravitinos. Also in this case we can overtake it by requiring that inflation dilutes away the gravitinos from the big-bang and by appropriately bounding the reheating temperature 34.

The second difficulty of this scenario comes from the low relic sneutrino abundance, due to the high $Z^{0}$ mediated annihilation cross section. However, this relic density increases in presence of an asymmetry between the sneutrinos and the antisneutrinos (this is possible since sneutrinos and antisneutrinos can be distinguished by their opposite lepton number). Indeed in this case almost only the sneutrinos (or the antisneutrinos) in excess survive the annihilation. $\square$ In this way one can consider the sneutrino relic abundance $Y_{\tilde{\nu}}$ as a free parameter dependent on the choice of the initial asymmetry.

The lifetime of this decay is given by 36.

$$
\tau=1.8 \cdot 10^{8} \sec \left(\frac{m_{3 / 2}}{10 \mathrm{GeV}}\right)^{2}\left(\frac{50 \mathrm{GeV}}{m_{\tilde{\nu}}}\right)^{5} .
$$

Consequently, the shift of $\lambda_{\text {eq }}$ is achieved for 1

$$
Y_{\tilde{\nu}}=\left(5.0 \cdot 10^{-9} \div \underline{2.4 \cdot 10^{-8}}\right)\left(\frac{10 \mathrm{GeV}}{m_{3 / 2}}\right)\left(\frac{m_{\tilde{\nu}}}{50 \mathrm{GeV}}\right)^{3 / 2}
$$

However, this value is too high, since the gravitinos produced by the decay would contribute to the critical density by

$$
\Omega_{3 / 2}=\frac{m_{3 / 2} Y_{\tilde{\nu}} s^{0}}{\rho_{\mathrm{cr}}^{0}} \simeq(90 \div \underline{70})\left(\frac{m_{\tilde{\nu}}}{50 \mathrm{GeV}}\right)^{3 / 2} .
$$

\footnotetext{
${ }^{14}$ This mass must also be kept high in order to avoid the decay $G \rightarrow \tilde{H} f \bar{f}$ via virtual higgs.

${ }^{15}$ The other annihilation channel $\tilde{\nu} \tilde{\nu} \rightarrow \nu \nu$ through zino exchange must also be suppressed by suitably rising the mass of the mediator.

${ }^{16}$ When we use this notation, the underlined value is referred to the $h=1$ case, while the other one to $h=0.4$.
} 
In Eq. (17) the dependence of $\Omega_{3 / 2}$ on the gravitino mass cancels out and one is left with the only free parameter $m_{\tilde{\nu}}$. Since $m_{\tilde{\nu}} \geq 43 \mathrm{GeV}$ [35] from accelerator experiments, the scheme here described cannot work because the produced gravitinos would overclose the universe.

This discussion shows that the realization of the shift of $\lambda_{\text {eq }}$ in an $R$ conserving supersymmetric scenario is problematic, although one has a priori the possibility to consider particles very low interacting and thus with long enough lifetimes. In particular, finding alternative schemes to the one involving gravitinos and axinos together (as discussed in [16]) appears a very difficult task.

\section{B. Broken $R$ parity}

In models with broken $R$ parity one may try to implement the shift of $\lambda_{\text {eq }}$ through the decay of the LSP. Indeed, one can consider very long lifetimes for this particle simply lowering enough the breaking of the $R$ symmetry.

If the breaking is made via one (or more) of the soft terms listed in Eq. (9), the decay typically leads to a nonnegligible production of photons or charged particles. As an example, if neutralino is the LSP and if one breaks $R$ through the term $\lambda_{i j k} L_{i} L_{j} E_{k}^{c}$, the process $\chi \rightarrow \nu e^{+} e^{-}$(through sneutrino or selectron exchange) constitutes the main decay channel. The same problem also arises in Gauge Mediated Supersymmetry Breaking (GMSB) models [37], where the LSP is typically an $m \sim \mathrm{keV}$ gravitino. In presence of soft breaking of $R$ parity the gravitino is very long living and it typically decays via loop into photons and neutrinos. We thus conclude that these schemes offer very little chances to implement the shift of $\lambda_{\text {eq }}$, since - as we said above - decays with the production of photons must be excluded.

A completely different and more workable scenario is offered by the possibility of breaking $R$ parity spontaneously. In the model described in [38] this is achieved by the extension of the MSSM with three isosinglet scalars $\square, \nu^{c}$, and $S$ carrying, respectively, lepton number (L) $0,-1$, and 1 .

In this model, the breaking of both global $L$ and $R$ symmetries gives birth to a massless goldstone boson $J$ - called majoron - given by the imaginary part of

$$
\frac{v_{l}^{2}}{V v^{2}}\left(v_{u} H_{u}-v_{d} H_{d}\right)+\frac{v_{l}}{V} \tilde{\nu}-\frac{v_{r}}{V} \tilde{\nu}^{c}+\frac{v_{s}}{V} \tilde{S}
$$

In the above equation the $H$ fields are the two higgs fields, $\tilde{\nu}$ is the sneutrino, $V=\sqrt{v_{r}^{2}+v_{s}^{2}}$,

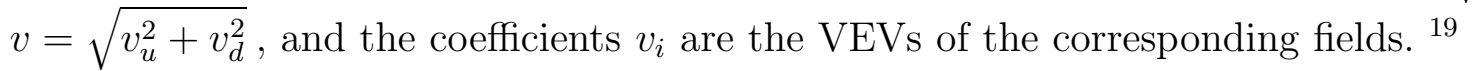

\footnotetext{
${ }^{17}$ If $R$ is broken by another of the terms in Eq. (9), also quarks are produced in the decay.

${ }^{18}$ The presence of all these new fields is necessary if one wants to break the lepton symmetry at tree level. However, if one allows for breaking through radiative corrections, the number of these singlets can be reduced 39 .
}

${ }^{19}$ We must choose $v_{l} \ll V$ for the majoron not to contribute to the invisible $Z^{0}$ width. 
If we take the neutralino as LSP, the breaking of $R$ parity enforces the process $\chi \rightarrow \nu_{L}+J$ as the main decay channel of $\chi$. The decay lifetime is given by 40]

$$
\tau=6 \cdot 10^{-26} \sec \alpha_{J}^{-1}\left(\frac{50 \mathrm{GeV}}{m_{\chi}}\right) \text {. }
$$

The coupling constant for this interaction $\left(\alpha_{J}=f^{2} / 4 \pi\right)$ depends on the mixing angles between the components of the neutralino that follow by diagonalizing the neutralino mass matrix [40]. In the present discussion we choose for definiteness $\chi=\tilde{Z}$. In this case we have

$$
\alpha_{J}=\frac{\alpha_{2}}{2 \cos ^{2} \vartheta_{W}} \frac{v_{l}^{2}}{v_{r}^{2}}
$$

We see from Eq. (19) that, in order to have have a late decay, a very tiny violation of $R$ parity is needed. Despite such a small value could seem rather unnatural, it is not inconceivable that it could arise as a residual effect of gravitation [40].

For $m_{\tilde{Z}} \simeq 50 \mathrm{GeV}$ the main annihilation channel of the zino is $\tilde{Z} \tilde{Z} \rightarrow f \bar{f}$ through sfermion exchange. This gives 41]

$$
m Y_{\tilde{Z}}=1.5 \cdot 10^{-7} \mathrm{GeV}\left(\frac{50 \mathrm{GeV}}{m_{\tilde{Z}}}\right)^{2}\left(\frac{m_{\tilde{f}}}{\mathrm{TeV}}\right)^{4}
$$

where $m_{\tilde{Z}}$ is the mass of the sfermions.

The shift of $\lambda_{\text {eq }}$ thus requires

$$
\tau=\left(1.3 \cdot 10^{8} \div \underline{2.9 \cdot 10^{9}}\right) \sec \left(\frac{m_{\tilde{Z}}}{50 \mathrm{GeV}}\right)^{4}\left(\frac{\mathrm{TeV}}{m_{\tilde{f}}}\right)^{8}
$$

Eqs. (4) and (5) can be "translated" into the bounds

$$
(250 \div \underline{5700}) \text { sec }<\tau<\left(8.6 \cdot 10^{12} \div \underline{5.0 \cdot 10^{12}}\right) \text { sec }
$$

on the zino lifetime. A more stringent upper bound comes from the non observation of the neutrinos, Eq. (6):

$$
\tau<2.6 \cdot 10^{10} \sec \left(\frac{50 \mathrm{GeV}}{m_{\tilde{Z}}}\right)^{2} .
$$

The requirement imposed by BBN are instead always satisfied for $m_{\tilde{Z}}$ not much greater than $50 \mathrm{GeV}$, since (see Fig. 2) 20

\footnotetext{
${ }^{20}$ for $m_{\tilde{Z}} \leq 50 \mathrm{GeV}$ and for $v_{r} \sim 100 \mathrm{GeV}$ we do not have to worry either about electromagnetic cascades produced by other decay channels of $\tilde{Z}$. Indeed, the branching ratio for the decays of the zino into photons or charged particles is 40

$$
\text { B.R. } \simeq 8 \cdot 10^{-5}\left(\frac{v_{r}}{100 \mathrm{GeV}}\right)^{2}\left(\frac{m_{\tilde{Z}}}{50 \mathrm{GeV}}\right)^{2} .
$$




$$
f_{\nu \rightarrow \gamma} \simeq 3 \cdot 10^{-3}\left(\frac{m_{\tilde{Z}}}{50 \mathrm{GeV}}\right)\left(\frac{10^{4} \mathrm{sec}}{\tau}\right) .
$$

We thus have the final bounds

$$
(250 \div \underline{5700}) \sec <\tau<2.6 \cdot 10^{10} \sec \left(\frac{50 \mathrm{GeV}}{m_{\tilde{Z}}}\right)^{2}
$$

which can be "translated" into the limit

$$
(0.5 \div \underline{0.8}) \mathrm{TeV}\left(\frac{m_{\tilde{Z}}}{50 \mathrm{GeV}}\right)^{3 / 4}<m_{\tilde{f}}<5 \mathrm{TeV}\left(\frac{m_{\tilde{Z}}}{50 \mathrm{GeV}}\right)^{1 / 2}
$$

for the sfermion mass, and into

$$
5 \cdot 10^{-18}\left(\frac{m_{\tilde{Z}}}{50 \mathrm{GeV}}\right)^{1 / 2}<f<\left(5 \cdot 10^{-14} \div \underline{1 \cdot 10^{-14}}\right)\left(\frac{50 \mathrm{GeV}}{m_{\tilde{Z}}}\right)^{1 / 2}
$$

for the coupling $f=\sqrt{4 \pi \alpha_{J}}$. From the smallness of $f$ in this allowed range, production of Majoron in stars [42,43], as well in laboratory experiments, result in unobservable effects.

\section{V. $M<$ MEV NEUTRINOS}

For light neutrinos the abundance is mass independent 44

$$
Y_{\nu}=3.88 \cdot 10^{-2}
$$

With this value, Eq. (3) is replaced by

$$
\left(\frac{m_{\nu}}{\mathrm{MeV}}\right)^{2}\left(\frac{\tau}{\mathrm{sec}}\right)=2.52 \cdot 10^{3} \div \underline{5.39 \cdot 10^{4}} .
$$

Since neutrinos are light and since we consider decays into invisible particles, we only have the general bounds set in Sec. II. We thus consider neutrinos with masses

$$
(15 \div \underline{93}) \mathrm{eV}<m_{\nu}<1 \mathrm{MeV}
$$

corresponding to lifetimes

$$
\left(2.5 \cdot 10^{3} \div \underline{5.4 \cdot 10^{4}}\right) \text { sec }<\tau<\left(\underline{6.2 \cdot 10^{12}} \div 1.1 \cdot 10^{13}\right) \text { sec } .
$$

In the following we apply this scheme to three different scenarios involving decaying neutrinos. For further details see also [45], where a more general discussion about decaying neutrinos (which however does not tackle the particular problem of the shift of the equivalence scale) is included.

\footnotetext{
${ }^{21} \mathrm{As}$ in the previous section, the underlined value is referred to the $h=1$ case, while the other one to $h=0.4$. In the case of a decaying neutrino we need a slightly greater amount of density energy in the decay, since in the thermal background there are only 2 neutrinos left [16].
} 


\section{A. Neutrinos in left-right symmetric models}

In models with left-right symmetry there exist natural isospin triplets which can mediate neutrino decay [46] (see Fig. 3i).

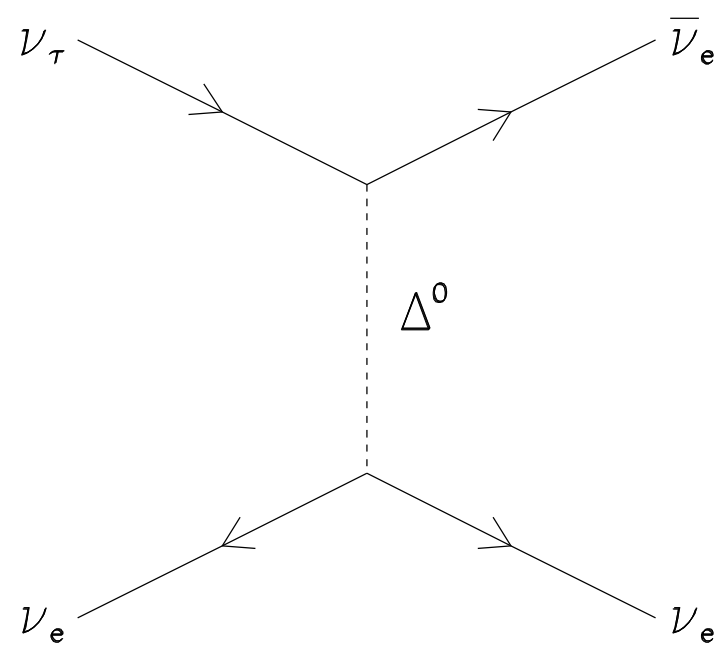

FIG. 3. Neutrino decay via isospin triplet.

The lifetime is given by

$$
\tau\left(\nu_{\tau} \rightarrow \nu_{e} \nu_{e} \bar{\nu}_{e}\right)=\left(\frac{m_{\Delta_{L}}^{0}}{m_{W}}\right)^{4}\left(\frac{m_{\mu}}{m_{\nu_{\tau}}}\right)^{5} u_{\nu_{\tau} \nu_{e}}^{-2} \cdot \tau\left(\mu \rightarrow \nu_{\mu} e \bar{\nu}_{e}\right)
$$

where $u_{\nu_{\tau} \nu_{e}}$ is the mixing between the two families and where the mass of the triplet is naturally of the order at which the left-right symmetry is broken [46:

$$
m_{\Delta_{L}}^{0} \simeq v_{r}>\mathrm{TeV}
$$

Defining $\epsilon=m_{W} / v_{r}$ we thus have $\epsilon<10^{-1}$. Furthermore it must be $\epsilon>10^{-4}$ or radiative decay would prevail [47. From Eq. (31) we get

$$
\left(\frac{m_{\mu}}{m_{\nu_{\tau}}}\right)^{5}=\left(\underline{1.98 \cdot 10^{-2}} \div 42.0\right)\left(\frac{\tau}{\mathrm{sec}}\right)^{5 / 2},
$$

and thus, from Eq. (33),

$$
\frac{\tau}{\sec }=\left(497 \div \underline{8.19 \cdot 10^{4}}\right)\left(u_{\nu_{\tau} \nu_{e}}\right)^{4 / 3} \epsilon^{8 / 3}
$$

If we limit ourselves to $m<\mathrm{MeV}$ neutrinos [and hence we take into account the lower bound set in Eq. (32)], even in case of maximal mixing $\left(u_{\nu_{\tau} \nu_{e}}=1\right)$ we have

$$
\tau>\left(2.5 \cdot 10^{3} \div \underline{5.4 \cdot 10^{4}}\right) \text { sec } \rightarrow \epsilon>1.8 \div \underline{0.9},
$$

which are above the allowed values. 


\section{B. Neutrinos in models with global symmetries}

We now consider a theory with a global symmetry spontaneously broken. The breaking induces the effective derivative coupling

$$
\Delta \mathcal{L}=V^{-1} j_{\mu} \partial^{\mu} \phi
$$

where $\phi$ is a (massless) goldstone scalar (or pseudoscalar) boson, $j_{\mu}$ is the current associated to the fermions which couple to the boson, and $V$ is the scale of symmetry breaking.

Due to the very weakly interaction of these bosons with the ordinary matter, decays $\nu \rightarrow \nu^{\prime} \phi$ have proven very feasible for the shift of the equivalence scale [17, 18]. Here we comment on two of the most popular types of goldstone bosons in which a heavy neutrino can decay into, i.e. familons and majorons.

Familons arise from the spontaneous breaking of an horizontal symmetry among the different generations of fermions 48 50]. The coupling familon-neutrinos can be written

$$
\Delta \mathcal{L}=V_{\nu^{\prime} \nu}^{-1} \bar{\nu}^{\prime} \gamma_{\mu} \nu \partial^{\mu} \phi
$$

If the natural assumption of universality between the couplings of the familon to neutrinos and to charged leptons is made, the scale of symmetry breaking must be taken $V>8$. $10^{10} \mathrm{GeV}$ in order to avoid problems with stellar energy loss due to familon bremsstrahlung of the electrons in the stellar medium [42]. 22]

Eq. (38) leads to the neutrino lifetime

$$
\tau\left(\nu \rightarrow \nu^{\prime} \phi\right)=3 \cdot 10^{6} \sec \left(\frac{V}{10^{10} \mathrm{GeV}}\right)^{2}\left(\frac{\mathrm{MeV}}{m_{\nu^{\prime}}}\right)^{3} .
$$

From Eq. (31) it follows

$$
\frac{V}{10^{10} \mathrm{GeV}}=(0.21 \div \underline{2.0})\left(\frac{\mathrm{sec}}{\tau}\right)^{1 / 4} .
$$

Limiting ourselves again to $m<\mathrm{MeV}$ neutrinos, from Eq. (32) we have

$$
1.3 \cdot 10^{-4} \div \underline{1.1 \cdot 10^{-3}}<\frac{V}{10^{10} \mathrm{GeV}}<0.03 \div \underline{0.13} .
$$

The upper limits are below the allowed value for $V$ stated above.

The stellar energy loss bound can be considerably weakened if we consider a goldstone boson which directly couples only to neutrinos. This is the case for the majoron [51] which arises in models with the spontaneous breaking of the lepton symmetry $(L)$.

\footnotetext{
${ }^{22}$ We are implicitly assuming that the lightest generation is involved in the family symmetry.
} 
The Gelmini-Roncadelli model [51] makes use of only left-handed neutrinos and has lepton number spontaneously broken by the $\mathrm{VEV}$ of an $\mathrm{SU}(2)_{\mathrm{L}}$ Higgs triplet. Nowadays this option is ruled out by the invisible width of $Z^{0}$.

We consider, instead, Majoron models à la Chikashige-Mohapatra-Peccei [51], with righthanded neutrinos and $L$ broken by the VEV of an isosinglet scalar $\phi$. This scalar carries $L=-2$ and is coupled to two right-handed neutrinos through a Yukawa interactions. When $\phi$ acquires a $\mathrm{VEV},\langle\phi\rangle=V$, L breaks and a Majorana mass term is recovered. In addition to this term, a Dirac mass term couples left-handed and right-handed neutrinos via the usual Yukawa interaction with the standard model higgs doublet. If $V$ is taken much bigger than the electroweak scale $M_{d}$, the diagonalization of the neutrino mass matrix splits the eigenvectors into heavy (mainly $\nu_{R}$, with mass of the order of $\mathrm{V}$ ) and light (mainly $\nu_{L}$, with mass of the order $M_{d}^{2} / V$ ) neutrinos (see-saw mechanism [52]).

However, this minimal model gives for the decay $\nu_{L} \rightarrow \nu_{L}^{\prime} \phi$ a lifetime bigger than the age of the universe, since the leading order term vanishes for flavor changing processes [47. This problem can be circumvented in non minimal models (see [53] and references therein). For natural values of the couplings, these models give lifetimes of the order [17]

$$
\tau\left(\nu \rightarrow \nu^{\prime} \phi\right)=6 \cdot 10^{4} \sec \left(\frac{V}{10^{10} \mathrm{GeV}}\right)^{2}\left(\frac{\mathrm{MeV}}{m_{\nu}}\right)^{3} .
$$

From Eqs. (31) and (42) we get

$$
\begin{aligned}
m_{\nu} & =(\underline{0.1} \div 2) \mathrm{keV}\left(\frac{\mathrm{V}}{10^{8} \mathrm{GeV}}\right)^{2} \\
\tau & =\left(4 \cdot 10^{8} \div \underline{4 \cdot 10^{12}}\right) \sec \left(\frac{10^{8} \mathrm{GeV}}{\mathrm{V}}\right)^{4} \\
M_{d} & =(\underline{3} \div 14) \mathrm{GeV}\left(\frac{\mathrm{V}}{10^{8} \mathrm{GeV}}\right)^{3 / 2} .
\end{aligned}
$$

The main bounds on the scale $V$ come in this case from majoron emission in supernovae explosions, and in particular from the neutrino pulse observation from SN1987A. Although this bounds depend on the model used and the discussion about them is not yet completely set [50.[2], from [54] we see that values $V>\mathrm{TeV}$ can be reasonably allowed. From Eq. (43) we see that scales $V$ about $\left(10^{8} \div 10^{9}\right) \mathrm{GeV}$ (values well above the SN limits) are suitable to obtain the shift of the equivalence scale.

\section{CONCLUSIONS}

In this paper we have considered several models with $\Omega_{\mathrm{CDM}+\text { baryons }}=1$ plus the presence of a late decaying particle in order to match the observed spectrum of fluctuations.

While having such late decaying particles is not unnatural in the most popular extensions of the standard model, our analysis shows that in most cases these models are severely constrained by cosmological and astrophysical considerations. The main reason is that in the scheme we are considering the decaying particle must dominate over the cold dark matter and the relic radiative background before it decays. Thus the decay products have 
high abundance and easily enter in conflict with bounds imposed, for example, by their non observation or by nucleosynthesis.

The stiffness of the problem becomes clear when one considers decays with the production of photons (or charged particles). In this case severe bounds force the photon energy density to be much less than the one needed to shift the equivalence scale. As a consequence, all the decays that give rise to the shift must have a negligible production of photons.

Less constrained but still delicate is the case when high energy neutrinos are produced. In addition to the bounds imposed by their non observation, we cannot neglect the possibility that they scatter off background thermal neutrinos, with consequent production of photons. We have seen that these bounds are strictly related to the energy of each neutrino. Thus - for equal amount of energy density of the produced neutrinos - bigger abundances and lower energies of the single particles are preferred.

Among the models studied, we focused in particular on supersymmetric extensions of the standard model. We have split the discussion in models with conserved and with broken $R$ parity.

In the case of conserved $R$ parity the main problems come from the necessity of delaying the decay time. Rather than fine tuning the masses (we saw that, in typical cases, masses must differ less than one part over $10^{13}$ ) we have studied models with very weakly coupled particles, like gravitinos or axinos. However, we saw that even in this case many difficulties arise.

If we instead choose to break $R$ parity, the natural candidate for the decaying particle is the LSP. Models where $R$ is explicitly broken via soft term can hardly accomplish the shift of the equivalence scale, since avoiding with the latter the production of photons or charged particles is very difficult. The situation improves in schemes where $R$ parity is spontaneously broken by the $V E V$ of an isosinglet, where decays like neutralino into neutrino and majoron can easily implement the shift of the equivalence scale.

Although we have presented particular processes in some detail, we have tried to maintain the discussion on the most possible general ground, underlying the main difficulties that each different class of models faces and (when possible) some ways to overcome them.

Given that evidences for $\Omega_{\text {matter }}<1$ need more solid confirmation, the option $\Omega_{\text {matter }}=1$ with a delayed equivalence scale remains still interesting. In case future observational work would favor this latter options, we hope that this work will be a basis for further more detailed investigations.

In particular, we think that important indications will come out from the observation of the acoustic peaks of the CBR [55]. An analysis of the peaks produced by the decays that we analyzed here could constitute an important complement to the present work.

\section{ACKNOWLEDGMENTS}

We are grateful to S. Borgani, E. Nardi, E. Pierpaoli, and A. Riotto for useful and lively discussions and to V.S. Berezinsky for interesting comments. In particular, we would like to thank S. Bonometto for pointing out some incorrectness in the first version of this paper. The work of D.M. is supported by an INFN Post-Doc fellowship. This work is 
partially supported by the EEC TMR network "Beyond the Standard Model", contract no. FMRX-CT96-0090.

[1] G.R. Blumenthal, S.M. Faber, J.R. Primack, and M. Rees, Nature 311, 517 (1984).

[2] M. Davis, G. Efstathiou, C.S. Frenk, and S.D.M. White, Nature 356, 489 (1992).

[3] K.B. Fisher. M. Davis, M.A. Strauss, A. Yahir, and J.P. Huchra, Astrophys. J 402, 42 (1993).

[4] J.A. Adams, G.G. Ross, and S. Sarkar, Phys. Lett. B 391, 271 (1997); Nucl. Phys. B 503, 405 (1997).

[5] D.S. Salopek, J.R. Bond, and J.M. Bardeen, Phys. Rev. D 40, 175 (1989).

[6] J. Legourgues, D. Polarski, and A.A. Starobinsky, astro-ph/9807019.

[7] Q. Shafi and F.W. Stecker, Phys. Rev. Lett. 53, 1292 (1984); S.A. Bonometto and R. Valdarnini, Astron. Astrophys. 146, 235 (1985); Astrophys. J. 299, L71 (1985); S. Achilli, F. Occhionero, and R. Scaramella, Astrophys. J. 299, 577 (1985); M. Davis, F.J. Summers, and D. Schlegel, Nature 359, 393 (1992); A.N. Taylor and M. Rowan-Robinson, Nature 359, 396 (1992); J.A. Holtsman and J.R. Primack, Astrophys. J. 405, 428 (1993).

[8] H. Pagels and J.R. Primack, Phys. Rev. Lett. 48, 233 (1982); J.R. Bond, A.S. Szalay and M.S. Turner, Phys. Rev. Lett. 48, 1636 (1982); S. Colombi, S. Dodelson, and L.M. Widrow, report no. FERMILAB-PUB-95-093-A, astro-ph/9505029.

[9] G. Efstathiou, W.J. Sutherland, and S.J. Maddlox, Nature 348, 705 (1990); A. Jenkins et al. (The Virgo Consortium), Astrophys. J. 499, 20 (1998).

[10] The Supernova Cosmology Project, S. Perlmutter et al., report no. LBNL-4-1801, astro$\mathrm{ph} / 9812133$.

[11] The High- $z$ Supernova Search Team, A.G. Riess et al., Astron. J. 116, 1009 (1998).

[12] R.R. Caldwell, R. Dave, and P.J. Steinhardt, Phys. Rev. Lett. 80, 1582 (1998); I. Zlatev, L. Wang, and P.J. Steinhardt, Phys. Rev. Lett. 82, 896 (1999).

[13] M.S. Turner, in the proceedings of the Nobel Symposium on Particle Physics and the Universe (Enkoping, Sweden, Aug. 1998), astro-ph/9901109.

[14] J. Mohr, B. Mathiesen, and G. Evrard, astro-ph/9901281; J. Carlstrom, Physica Sripta, in press.

[15] J. Bond and G. Efstathiou, Phys. Lett. B 265, 245 (1991).

[16] H.B. Kim and J.E. Kim, Nucl. Phys. B 433, 421 (1995).

[17] M. White, G. Gelmini, and J. Silk, Phys. Rev. D 51, 2669 (1995).

[18] H. Kikuchi and E. Ma, Phys. Rev. D 51, 296 (1995); A.D. Dolgov, S. Pastor, and J.W.F. Valle, report no. FTUV/95-14, IFIC/95-14, astro-ph/9506011.

[19] M.S. Turner and J.A. Tyson, Rev. Mod. Phys. 71, S145 (1999).

[20] E. Lisi, S. Sarkar, and F.L. Villante, Phys. Rev. D 59, 123520 (1999).

[21] J. Ellis, G.B. Gelmini, J.L. Lopez, D.V. Nanopoulos, and S. Sarkar, Nucl. Phys. B 373, 399 (1992).

[22] P. Gondolo, G. Gelmini, and S. Sarkar, Nucl. Phys. B 392, 111 (1993).

[23] V.S. Berezinsky, Nucl. Phys. B 380, 478 (1992).

[24] W. Zhang et al. (Kamiokande II collaboration), Phys. Rev. Lett. 61, 385 (1988). 
[25] W. Fulgione (LVD collaboration) in the proceedings of "TAUP 97", fifth International Workshop on Topics in Astroparticle and Underground Physics (Gran Sasso, Sep. 1997), Nucl. Phys. B Proc. Suppl. 70, 123 (1999), p. 469.

[26] Y. Fukuda et al. (SuperKamiokande collaboration), Phys. Rev. Lett. 82, 1810 (1999); R. Barbieri, G. Fiorentini, G. Mezzorani, and M. Moretti, Phys. Lett. B 259, 119 (1991).

[27] M. Kawasaki and T. Moroi, Phys. Lett. B 346, 27 (1995).

[28] J. Gratsias, R.J. Scherrer, and D.N. Spergel, Phys. Lett. B 262, 298 (1991).

[29] E. Holtmann, M. Kawasaki, K. Kohri, and T. Moroi, Phys. Rev. D 60, 023506 (1999).

[30] R.J. Protheroe, T. Stanev, and V.S. Berezinsky, Phys. Rev. D 51, 4134 (1995).

[31] G. Jungman, M. Kamionkowski, and K. Griest, Phys. Rept. 267, 195 (1996).

[32] V. Berezinsky, A.S. Joshipura, and J.W.F. Valle, Phys. Rev. D 57, 147 (1998).

[33] J.A. Frieman and G.F. Giudice, Phys. Lett. B 224, 125 (1989).

[34] M. Bolz, W. Buchmüller, and M. Plümacher, Phys. Lett. B 443, 209 (1998).

[35] J. Ellis, T. Falk, K.A. Olive, and M. Schmitt, Phys. Lett. B 413, 355 (1997).

[36] T. Moroi, H. Murayama, and M. Yamaguchi, Phys. Lett. B 303, 289 (1993); E. Pierpaoli, S. Borgani, A. Masiero, and M. Yamaguchi, Phys. Rev. D 57, 2089 (1998).

[37] P. Fayet, Phys. Lett. B 84, 421 (1979); 175, 471 (1986).

[38] A. Masiero, J.W.F. Valle, Phys. Lett. B 251, 273 (1990).

[39] G.F. Giudice, A. Masiero, M. Pietroni, and A. Riotto, Nucl. Phys. B 396, 243 (1993).

[40] V. Berezinsky, A. Masiero, and J.W.F. Valle, Phys. Lett. B 266, 382 (1991).

[41] J. Ellis, J.S. Hagelin, D.V. Nanopoulos, K. Olive, and M. Srednicki, Nucl. Phys. B 238, 453 (1984).

[42] G.G. Raffelt "Stars as Laboratories for Fundamental Physics" (The University of Chicago Press, 1996).

[43] D. Dearborn, D.N. Schramm, G. Steigman, Phys. Rev. Lett. 56, 26 (1986).

[44] E.W. Kolb and M.S. Turner, "The Early Universe" (Addison-Wesley Publishing Company, 1990).

[45] J.W.F. Valle, Prog. Part. Nucl. Phys. 26, 91 (1991).

[46] M. Roncadelli and G. Senjanović, Phys. Lett. B 107, 59 (1981).

[47] J. Schechter and J.W.F. Valle, Phys. Rev. D 25, 774 (1982).

[48] F. Wilczek, Phys. Rev. Lett. 49, 1549 (1982).

[49] D.B. Reiss, Phys. Lett. B 115, 217 (1982); G.B. Gelmini, S. Nussinov, and T. Yanagida, Nucl. Phys. B 219, 31 (1983).

[50] G.B. Gelmini, S. Nussinov, and R.D. Peccei, Int. J. Mod. Phys. A 7, 3141 (1992).

[51] Y. Chikashige, R.N. Mohapatra, and R.D. Peccei, Phys. Lett. B 98, 265 (1981); Phys. Rev. Lett. 45, 1926 (1980); G.B. Gelmini and M. Roncadelli, Phys. Lett. B 99, 411 (1981); H. Georgi, S.L. Glashow, and S. Nussinov, Nucl. Phys. B 193, 297 (1981).

[52] M. Gell-Mann, P. Ramond, and R. Slansky, in the proceedings of "Supergravity", Stony Brooke, New York, 1979, ed. by P. Niuwenhuizen and D. Freedman (Amsterdam, NorthHolland, 1979), p. 315; T. Yanagida, in the proceedings of the Workshop on "Unified Theories and the Baryon Number of the Universe", Tsukuba, Ibaraki, Japan, 1979, ed. by O. Sawada and A. Sugamoto (KEK report 79-18, Tsukuba, Japan, 1979), p. 95.

[53] G.B. Gelmini and E. Roulet, Rept. Prog. Phys. 58, 1207 (1995).

[54] K. Choi and A. Santamaria, Phys. Rev. D 42, 293 (1990).

[55] E. Pierpaoli and S.A. Bonometto, report no. SISSA-55-98-A, astro-ph/9806037. 